\title{
Severe Periodontal Disease Manifested in Chronic Disseminated Type of Langerhans Cell Histiocytosis in a 3-Year Old Child
}

\author{
${ }^{1}$ Monika Bansal, ${ }^{2}$ Vinay Kumar Srivastava, ${ }^{3}$ Rajesh Bansal, ${ }^{4}$ Vineeta Gupta, ${ }^{5}$ Manish Bansal, ${ }^{6}$ Shashikant Patne
}

\begin{abstract}
Langerhans cell histiocytosis ( $\mathrm{LCH})$, previously known as histiocytosis $\mathrm{X}$, is a rare idiopathic disorder of reticulo-endothelial system with abnormal proliferation of bone marrow derived Langerhans cells along with a variable number of leukocytes, such as eosinophils, neutrophils, lymphocytes and plasma cells. Three years old male child presented with multifocal osteolytic lesions and papulosquamous skin lesions. Clinical and radiographic features, such as severe alveolar bone loss, mobility of teeth, precocious eruption of teeth, floating appearance of teeth in orthopantomogram (OPG), osteolytic lesion in skull and cutaneous lesions were highly suggestive of $\mathrm{LCH}$ disease. Skin biopsy confirmed a diagnosis of LCH. Induction chemotherapy with oral prednisolone and intravenous vinblastine was started. Child responded well to chemotherapy. The clinical significance of the presented case is to diagnose the case of $\mathrm{LCH}$ on the basis of the manifestation of severe periodontal disease as this can be first or only manifestation of $\mathrm{LCH}$. A dentist plays a major role in the multidisciplinary treatment of $\mathrm{LCH}$ through routine examination and periodic follow-up.
\end{abstract}

Keywords: Floating teeth, Langerhans cell histiocytosis, Osteolytic lesion, Seborrheic dermatitis.

How to cite this article: Bansal M, Srivastava VK, Bansal R, Gupta V, Bansal M, Patne S. Severe Periodontal Disease Manifested in Chronic Disseminated Type of Langerhans Cell Histiocytosis in a 3-Year Old Child. Int J Clin Pediatr Dent 2014;7(3):217-219.

\footnotetext{
${ }^{1,5,6}$ Assistant Professor, ${ }^{2,4}$ Associate Professor, ${ }^{3}$ Reader

${ }^{1-3}$ Department of Dental Sciences, Institute of Medical Sciences, Banaras Hindu University, Varanasi, Uttar Pradesh India

${ }^{4}$ Department of Pediatrics, Faculty of Modern Medicine, Institute of Medical Sciences, Banaras Hindu University, Varanasi, Uttar Pradesh, India

${ }^{5}$ Department of Skin, Faculty of Modern Medicine, Institute of Medical Sciences, Banaras Hindu University, Varanasi, Uttar Pradesh, India

${ }^{6}$ Department of Pathology, Faculty of Modern Medicine, Institute of Medical Sciences, Banaras Hindu University, Varanasi, Uttar Pradesh, India
}

Corresponding Author: Monika Bansal, Assistant Professor Department of Dental Sciences, Institute of Medical Sciences Banaras Hindu University, Varanasi, Uttar Pradesh, India e-mail: dr.monika97@rediffmail.com

\section{Source of support: Nil}

Conflict of interest: None

\section{INTRODUCTION}

Langerhans cell histiocytosis is a group of idiopathic disorders of reticuloen-dothelial system characterized by abnormal proliferation of bone marrow derived Langerhans cells. ${ }^{1}$ Abnormal proliferation of these cells replaces the bone and invades into the skin, mucosa and internal organs leading to tissue destruction. Langerhans cell histiocytosis was formerly known as histiocytosis $X^{2}$ The term histiocytosis denotes the proliferation of histiocytes and other inflammatory disorders and the letter $X$ represents the unknown etiology of the disease. However, recently the terminology has changed to $\mathrm{LCH}$ or class I histiocytosis instead of histiocytosis X due to the fact that histiocytes are similar to the Langerhans cells present in the skin and mucosa. ${ }^{3}$ Langerhans cell histiocytosis is classified into three clinical forms depending upon the age and clinical presentation: (a) chronic localized form which includes unifocal or multifocal radiolucencies of bones and known as eosinophilic granuloma, (b) chronic disseminated form also known as Hand-Schuller-Christian disease and (c) acute disseminated form also called as Letterer-Siwe disease. ${ }^{4}$ Langerhans cell histiocytosis can have an extremely variable presentation which can present difficulty in diagnosis. Our objective is to focus on the importance of changes in the periodontal tissues in a 3 years old male child having chronic disseminated type of LCH disease.

\section{CASE REPORT}

A 3-year old male child reported at the Dental Outpatient Department, University Hospital, Varanasi, with the complaints of rapid loss of teeth for 7 months and difficulty in chewing food. On general examination, child was active with stable vitals and a short stature. A cervical lymph node was palpable. Liver and spleen were within normal range. Bilateral fine crepts were present on chest examination. Seborrheic dermatitis like papulosquamous lesions were present on the scalp, neck and shoulder region. Few hypopigmented macules were 


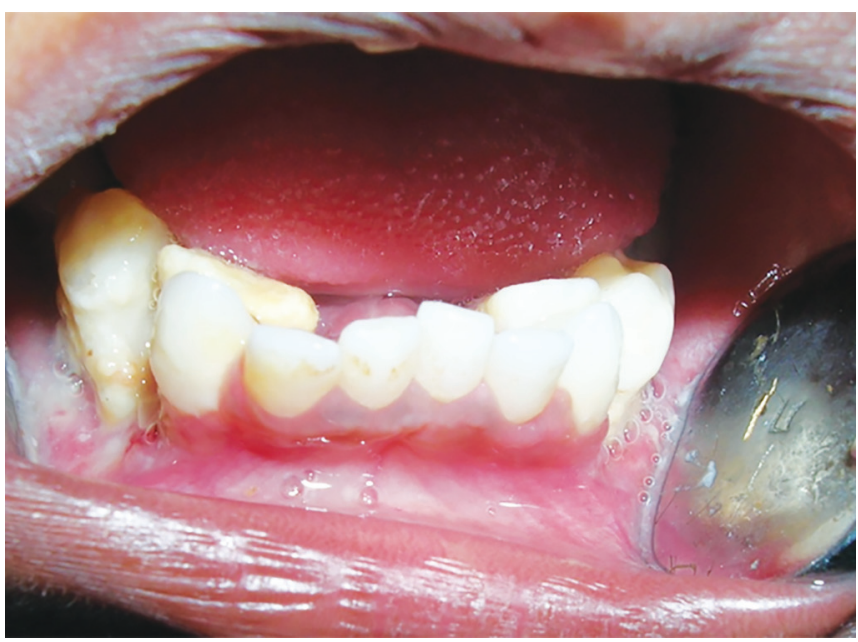

Fig. 1: Intraoral examination showed generalized severe periodontitis
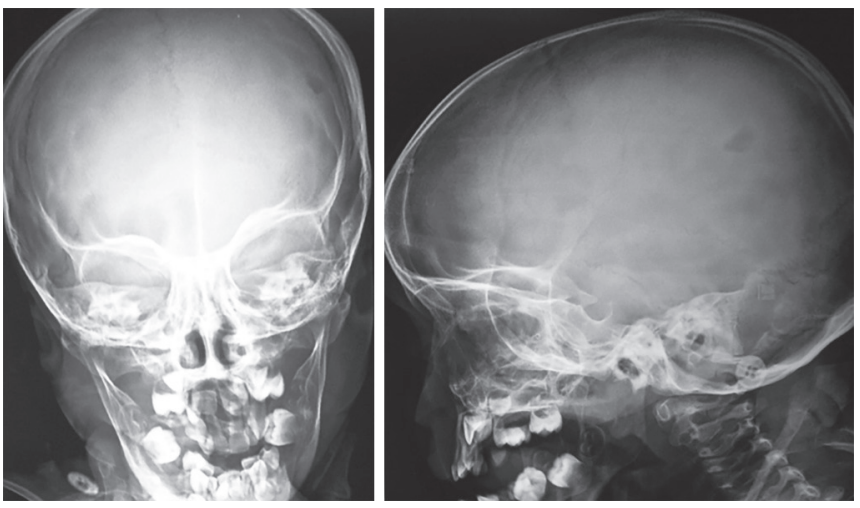

Fig. 3: PA view of skull revealed the osteolytic lesion

also present over the back and face. Nails of hand were deformed and showed atrophy. On intraoral examination, all deciduous teeth except maxillary right second molar, left first and second molar and precocious eruption of permanent first molars in all quadrants and both permanent mandibular lateral incisors were present. Poor oral hygiene, bleeding on probing and generalized severe periodontitis in the form of gingival recession was present (Fig. 1). All present deciduous posterior teeth as well as premature permanent teeth had grade III mobility. Complete blood count, thyroid profile, liver function test (LFT), Elisa for serotesting of human immunodeficiency virus (HIV), OPG X-ray, X-ray skull, $\mathrm{X}$-ray chest and abdominal ultrasound were advised. Hemoglobin was 9.5 $\mathrm{gm} / \mathrm{dl}$ with normal total and differential count. Thyroid function was within normal limits. LFT was normal except elevated alkaline phosphatase which was $1191 \mathrm{U} / \mathrm{L}$. HIV test was nonreactive. OPG X-ray revealed multiple radiolucent lesions and floating teeth in the posterior region of maxilla and mandible due to severe alveolar bone loss and premature erupted permanent teeth do not have their roots (Fig. 2). X-ray of skull revealed multiple osteolytic lesions (Fig. 3). X-ray chest and abdominal ultrasound did not show any significant finding. After

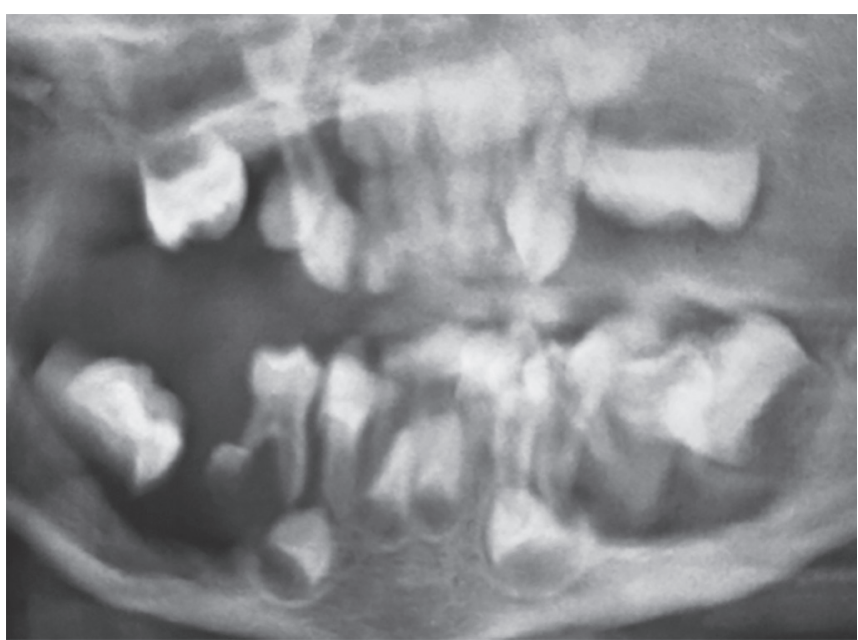

Fig. 2: OPG X-ray revealed multiple radiolucent lesions and floating teeth

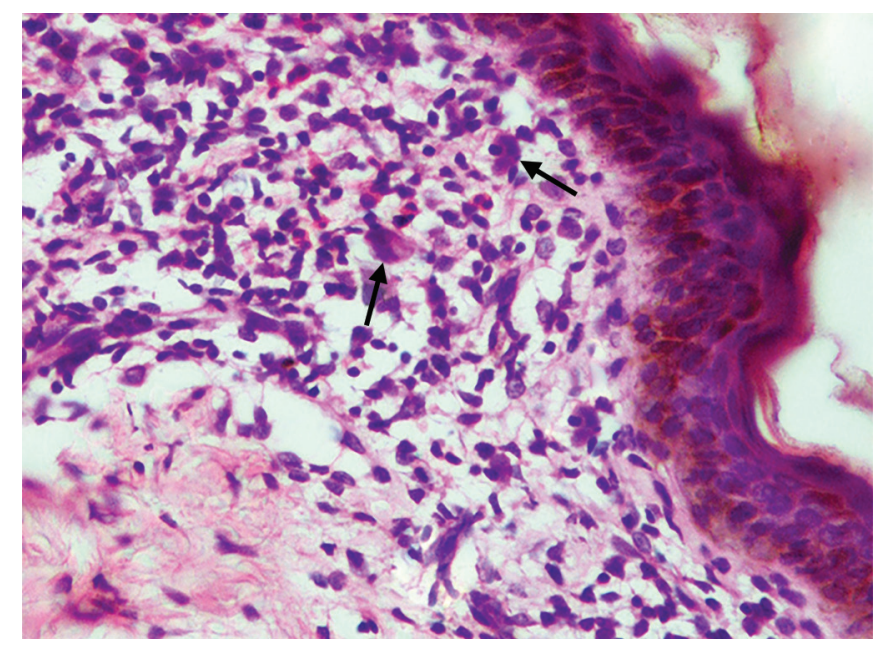

Fig. 4: H\&E staining at 400x showed Langerhans cells (arrows) and dense aggregates of inflammatory cells in superficial dermis comprising of histiocytes, lymphocytes, eosinophils and plasma cells

clinical, laboratory and radiographical examination, all features were suggestive of LCH and biopsy taken from one of the papulosquamous lesions present over the scalp was sent for histopathological examination. Histopathologic examination showed proliferation of langerhans cells and aggregates of inflammatory cells comprising of histiocytes, lymphocytes, plasma cells and eosinophils (Fig. 4). Occasional langerhans cells showed numerous grooves and folds with abundant cytoplasm. The skin biopsy findings were diagnostic of $\mathrm{LCH}$. Induction chemotherapy was started with weekly vinblastine and oral predisolone for 6 weeks followed by maintenance chemotherapy. Child showed good response and is in regular follow-up. Temporary prosthesis was also planned for replacement of missing teeth.

\section{DISCUSSION}

The clinical presentation of LCH is variable and it mostly affects the skin and mucosa in the form of seborrheic dermatitis like lesions on face and scalp along with 
internal organ involvement. Involvement of teeth is an uncommon finding and rarely a presenting feature in most of the cases.

In the present case, clinical features of periodontal disease such as bleeding on probing, gingival recession, severe alveolar bone loss, and mobility of teeth in 3 years old child make the diagnosis difficult and it may be misdiagnosed as early onset periodontal disease. But other clinical and radiographic features such as precocious eruption of teeth, floating appearance of teeth in OPG X-ray, skull osteolytic lesion and skin lesions were suggestive of $\mathrm{LCH}$ disease. The oral manifestations may be the first sign of LCH disease and premature loss of deciduous teeth with bone loss and floating teeth is a clear sign of LCH disease. ${ }^{5}$ The present case was a case of multifocal single system due to presence of only bony lesions and skin lesions. In multifocal multisystem, LCH there is involvement of skeletal system, liver, spleen, hematopoietic system and lungs. Bony lesions were present in the X-ray of skull, maxilla and mandible and confirm the skeletal system involvement. Abdominal ultrasound, chest X-ray and complete blood count did not show any significant findings and excluded the involvement of liver, spleen, lung and bone marrow. A low $\mathrm{Hb}$ alone with normal TLC and platelet count can be because of iron deficiency anemia and will not classify as bone marrow involvement.

After clinical and radiographic findings, the $\mathrm{LCH}$ disease was confirmed on histopathologic examination of skin biopsy. The cell membrane of the histiocytes in LCH disease have CD1a antigen. ${ }^{6}$ Electron microscopy is also helpful in making the definitive diagnosis of $\mathrm{LCH}$ by identifying the specific marker known as 'Birbeck granules' in the cytoplasm of histiocytes. These granules possess tennis-racket morphology with transverse striations. ${ }^{7}$

Although the disease can present at any age but more than $50 \%$ of LCH cases are found in under the age of 10 years. ${ }^{6} \mathrm{LCH}$ is most commonly found in males. The incidence of $\mathrm{LCH}$ disease ranges from 0.5 to 5.4 cases per million persons per year. ${ }^{8}$ The etiology and pathogenesis is still unknown. Various theories have been proposed regarding the possible etiology including immunologic reactions, viruses, bacteria or genetic involvement. ${ }^{9-11}$
Treatment of LCH includes surgery, radiotherapy, chemotherapy and steroid therapy alone or in combination depending upon the severity and extent of the disease. ${ }^{12}$ The present case was chronic disseminated type of $\mathrm{LCH}$; therefore, chemotherapy was started including oral prednisolone and vinblastine intravenously. Patient has remained in regular follow-up.

The clinical significance of the presented case is that the severe periodontal disease may help in the diagnosis of LCH as these can be first or only manifestation of LCH and the dentist can play a major role in the multidisciplinary treatment of $\mathrm{LCH}$.

\section{REFERENCES}

1. Watanabe K. Prepuberal periodontitis: a review of diagnostic criteria and differential diagnosis. J Periodont Res 1990;25(1): 31-48.

2. Pereda CMM, Rodriguez VG, Moya BG, Garcia CM. Langerhans cell histiocytosis: literature review and descriptive analysis of oral manifestation. Med Oral Pathol Oral Cir Bucal 2009;14(5):222-228.

3. Rapidis AD, Langdon JD, Harvey PW, Patel MF. Histiocytosis X: an analysis of 50 cases. Int J Oral Surg 1978;7(2):76-84.

4. Wood NK, Goaz PW. Differential diagnosis of oral and maxillofacial lesions: multiple, well-defined radiolucencies. 5 th ed. Mosby publishing, USA; 1998. p. 380-392.

5. Shirley JC, Thornton JB. Oral manifestations of Langerhans cell histiocytosis: review and report of case. ASDC J Dent Child 2000;67(4):293-296.

6. Neville BW, Damm DD, Allen CM, Bouquot JE. Oral and maxillofacial pathology: haematologic disorder. 2nd ed. New Delhi: Elsevier Publishing; 2008. p. 513-515.

7. Lombardi T, Hauser C, Budtz-Jorgensen E. Langerhans cells: structure, function and role in oral pathological conditions. J Oral Pathol Med 1993;22(5):193-202.

8. Alston RD, Tatevossian RG, Mcnally RJ, Kelsey A, Birch JM, Eden TO. Incidence and survival of childhood langerhans cell histiocytosis in northwest England from 1954 to 1998. Pediatr Blood Cancer 2007;48(5):555-560.

9. Hernandez-Juyol M, Boj-quesada JR, Gallego Melcon S. Oral menifestations of Langerhans cell histiocytosis: case study of 2-year-old boy. Med Oral 2003;8(1):19-25.

10. Hartman KS. Histiocytosis X: a review of 114 cases with oral involvement. Oral Surg Oral Med Oral Pathol 1980;49(1):38-54.

11. Egeler RM, D'Angio GJ. Langerhans cell histiocytosis. J Pediatr 1995;127(1):1-11.

12. Roy Choudhary A, Shah N, Prakash H, Mukhopadhay S, Chopra P. Eosinophilic granuloma of the jaws. Br J Oral Maxillofac Surg 1998;36(5):380-383. 